\title{
Lymph node disease with lymphocytic abnormal chromatin clumping in a myelodysplastic/myeloproliferative syndrome
}

\author{
J Gardais
}

\begin{abstract}
A case of abnormal chromatin clumping (ACC) which arose during the course of a myelodysplastic/myeloproliferative syndrome is described in a 61 year old woman who died of haemorrhage 43 months after diagnosis. Mature granulocytes exhibited the same nuclear abnormality described in other patients reported. Unusually, she presented with advanced splenomegaly and lymphadenopathy. This case was the third example of ACC in lymphocytes, the first with clinically confirmed lymphadenopathy.

Diagnosis of this subset can be based on: older age; short duration of symptoms; no specific karyotypic damage; non-rearranged bcr; proliferative growth pattern in vitro; numerous circulating myelocytes; profound thrombocytopenia.
\end{abstract}

(F Clin Pathol 1993;46:177-179)

A new variant of myelodysplastic syndrome has recently been described. It comprises concomitant cytopenias, proliferative features, and, in particular, abnormal clumping of chromatin (ACC) of certain mature granulocytes. This morphological characteristic has also been observed in medullary or circulating lymphocytes in only two patients. ${ }^{1}$ We report an additional case with the same abnormality in lymphocytes within a lymphadenopathy, a rarely reported complication.

\section{Case report}

A 61 year old woman presented in March 1987 with the following peripheral blood picture haemoglobin: $96 \mathrm{~g} / 1$ mean corpuscular volume (MCV) $93.9 \mathrm{fl}$, reticulocytes $37.5 \times 10^{9} / \mathrm{l}$, anisocytosis, poikilocytosis, a white cell count of $3.3 \times 10^{9} / 1$ with a normal differential, and a platelet count of $51 \times 10^{9} / 1$. She was otherwise entirely well and there were no relevant medical or family histories.

Physical examination indicated no abnormality apart from pallor. Serum $B_{12}$, serum and red cell folate, and serum iron concentrations were all normal as were a biochemical screen and neoplastic markers. There was a polyclonal increase in immunoglobulin concentrations. A bone marrow aspirate showed erythroid hyperplasia without sideroblastosis with increased cellularity and $15 \%$ blasts. It was considered to be morphologically consistent with non-sideroblastic refractory anaemia with excess of blasts. She received low dose aracytine treatment which was discontinued after only four days because of the sudden appearance of a severe thrombocytopenia and a fall in haemoglobin concentration to $60 \mathrm{~g} / 1$. It was felt that simple supportive treatment would be appropriate and she was maintained on regular blood transfusions. In July 1987 a further bone marrow aspirate showed a reduced blastosis.

She was admitted again in February 1988 with $10 \%$ blasts in the peripheral blood. A repeat bone marrow examination showed $24 \%$ blasts. Seven days of intravenous aclacinomycin and oral thioguanine was started. Despite clinical improvement she did not achieve complete remission. Hydroxyurea $500 \mathrm{mg} /$ day and thioguanine $50 \mathrm{mg} / \mathrm{day}$, then $25 \mathrm{mg} /$ day were given. During follow up, however, her anaemia and thrombocytopenia persisted despite deferred chemotherapy; the white cell count rose to $14.7 \times 10^{9} / 1$ with $6.5 \%$ blasts, $6.5 \%$ promyelocytes, $31 \%$ myelocytes, $18 \%$ metamyelocytes, $10.5 \%$ monocytes, $12.5 \%$ neutrophils and $15 \%$ lymphocytes in April 1988. Chronic myelomonocytic leukaemia (CMML) was diagnosed. The haematological picture over the subsequent period was characterised by a slow progressive increase in white cells and the persistence of monocytosis, immature granulocytic cells, and blastosis in the peripheral blood.

In October 1989 a repeat bone marrow aspirate disclosed an increase in blast cells to $37 \%$ with abnormalities of mature granulocytes (fig 1). Neutrophils and metamyelocytes were characterised by chromatin clumping into large blocks separated by clear zones, mimicking nuclear fragmentation and nuclear membrane disruption. These abnormalities were not seen in myelocytes and promyelocytes. Degranulation was also observed in mature forms. Moderate dyserythropoiesis was observed but megakaryocytes were absent. A slight monocytosis suggested the diagosis of CMML in transformation. Chromosomal analysis of bone marrow cells, using synchronisation and $\mathbf{R}$ banding, showed a 46XX karyotype in five metaphases examined. Profound thrombocytopenia persisted, and between January 1990 and July-August 1990 the white cell counts fluctuated from 44 to $68.7-116 \times 10^{9} / 1$ with 6,18 , and $23 \%$ blasts and 4,23 , and $9 \%$ monocytes, respectively. There was no perceptible response to danazol. Meanwhile, the patient developed a progressive splenomegaly and cervical lymphadenopathies. A cytogram of the latter exhibited the same abnormal mature granulocytes found 


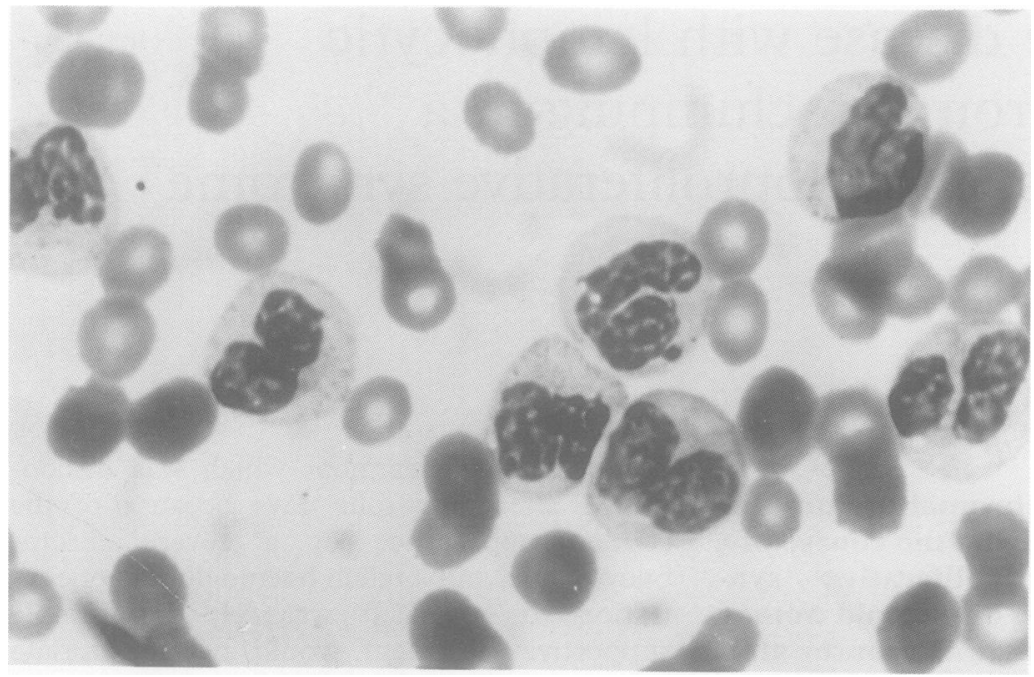

Figure 1 Mature granulocytes in peripheral blood presenting large blocks of chromatin separated by clear zones (May-Grünwald-Giemsa).

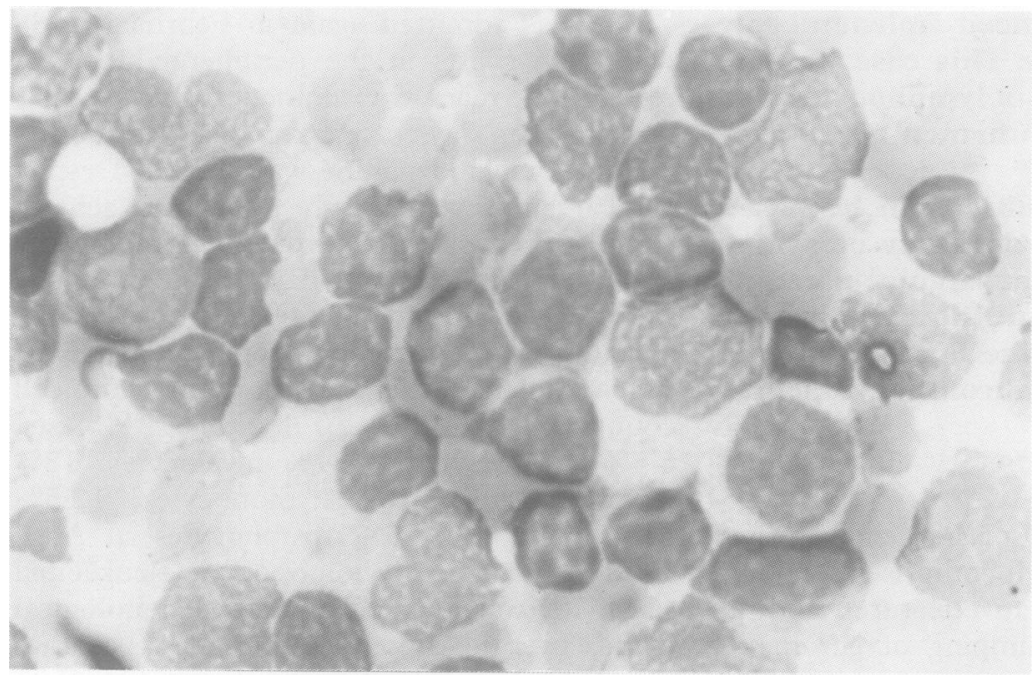

Figure 2 Metaplasia of lymph node with lymphocytes exhibiting the same ACC as in mature granulocytes (May-Grünwald-Giemsa).

in bone marrow; the lymphocytes were characterised by the similar ACC (fig 2). Because of severe thrombocytopenia and allogen immunisation to platelet concentrates, chemotherapy was deferred. However, her spleen increased in size, repeated bleedings required numerous blood transfusions and, in October 1990, she died 43 months after diagnosis with signs of digestive haemorrhage. Death occurred only one year after the diagnosis of ACC had been made. Postmortem examination was not performed.

\section{Discussion}

The nature of ACC has been discussed before. DNA analysis failed to reveal any hyperploid clone in two previous cases. Therefore, for Felman, ${ }^{1}$ this clumping does not correspond to chromatin increase, but is probably related to an abnormal distribution within the nucleus and a modification of the heterochromatin: euchromatin ratio. No systemic karyotypic abnormality was found in the same study. In another report the karyotype was also normal and no consistent rearrangement of the $b c r$ was identified after isolation of high molecular weight DNA and Southern blotting analysis from the patient's peripheral blood. ${ }^{2}$ As pointed out by Brizard, ${ }^{3}$ we cannot argue against a case of "atypical chronic myelocytic leukemia (CML)" where non-rearranged $b c r$ identification was obviously demonstrated. ${ }^{4}$

The case reported here resembles all the other 19 described to date in several respects. ${ }^{125-9}$ ACC was seen mainly in neutrophils, band forms and metamyelocytes. Myelodysplastic features were also evident. ACC was absent at diagnosis and examination of lymphocytes contrasted with later abnormalities. However, ACC appeared within lymphadenopathy during the course of the disease. Such a clinical complication has already been reported in CMML. ${ }^{11}$ Lymphadenopathy was rarely recognised in $\mathrm{CML}$ many years ago. ${ }^{12}$ Until now, ACC in leucocytes had been identified from haematological criteria among either myelodysplastic syndromes (MDS), or "atypical CML", closely related to true CML and CMML. That this feature has been reported in an acute phase of $\mathrm{CML}^{7}$ and that the well known CMML has been included in MDS by the FAB group ${ }^{13}$ is confusing. In one of the two cases previously described by Gustke, ${ }^{6}$ where lymphocytes had no abnormal chromatin, metaplasia of the spleen, liver, and lymph nodes at the time of a splenectomy was observed.

Evidence of the underlying clonality of haematopoiesis in MDS has been established by cytogenetic, glucose 6-phosphate dehydrogenase (G6PD), or, more recently, restriction fragment length analysis. ${ }^{14-18}$. Prchal et al postulated that the clonal abnormality in myelodysplasia involves a stem cell precursor of both myeloid and lymphoid cell lines. ${ }^{15}$ This is supported by investigation of a patient with myelodysplasia who was heterozygous for G6PD; the same isoenzyme was present in all haematopoietic cells, including lymphocytes. Nowadays, there is a general agreement concerning the clonal nature of all malignant blood disorders, resulting from neoplastic transformation of a pluripotent stem cell, regardless of morphological subtypes. Consequently, it raises the possibility of lymphoid lineage involvement. This "unitary theory" is sustained by a spectrum of several remarkable studies, clinical observations, or unique associations: Wallis and Joyner described a patient with chronic lymphocytic leukaemia (CLL) untreated for five years who subsequently developed acute myeloid leukaemia. ${ }^{19}$ These authors pointed out that this secondary disease may occur as a result of reduced immune competence consequent on CLL. Several examples of associations between myeloid disturbances, as observed in MDS, and immune dysfunction have been published. Acute lymphoblastic leukaemia following $\mathrm{MDS}^{2021}$ association of lymphoma, ${ }^{22}$ or immunological abnormalities $^{2324}$ with MDS support the concept of lymphocyte down-regulation of a myoloid clone. Bastion et al also described a patient in whom MDS coexisted with CLL for more than nine years. ${ }^{25}$ The patient's serum inhibited the patient's own granulocyte-macrophage colony forming units. Furthermore, $\mathrm{T}$ cell 
receptor $\delta$ gene rearrangement has been shown in CMML. ${ }^{26}$ On the other hand, unstable and reversible (to myeloid cells) differentiation has been found in mature $T$ lymphoid leukaemia cells. ${ }^{27}$ In our case ACC in lymphocytes within a lymphadenopathy would provide an additional argument supporting the fact that, in MDS or related disorders, progenitor cells are capable of clonal differentiation along a variety of pathways. The recombination events required for gene rearrangement demand that the chromatin be "open" and accessible to recombinant enzymes ${ }^{28}$ at the appropriate phase of the developmental cycle. Thus an imbalance in the distribution of the two chromatins could sometimes exceptionally be found at microscopic level, as in our case and other ACC examples. Lymphadenopathy with lymphocytes exhibiting ACC is a new characteristic which contributes to support the view that this particular myelodysplastic/ myeloproliferative syndrome is a homogenous group of disorders with, overall, poor prognosis $^{29}$ among heterogeneous subsets of multipotent stem cell derived blood diseases. This syndrome is placed precisely between myelodysplastic and myeloproliferative ones and is characterised by the coexistence of some mixed features suggesting both. It represents an atypical and rare expression of a lingering multiphasic panmyelopathy that involves all products of the marrow stem cell. ${ }^{30}$ It perhaps indicates that the same marrow insult can manifest itself in several ways and that an initial oncogenic event selects a clone of stem cells, which retain the capacity to differentiate into both lineages, these being marked by functional abnormalities. One of them exhibits chromatin disturbance, accounting for a new evidence of a common lymphoid and myeloid progenitor cell. However, whether ACC in lymphoid cells in lymphadenopathy in our patient rather represents proof of the common stem cell origin of two myeloid and lymphoid haematological manifestations or a morphological consequence of immune dysregualtion remains to be determined.

In conclusion, our patient's clinical course and haematological symptoms complete the definition of this newly described subset. Finally, the diagnosis of this syndrome is based on the following: older age; short duration; no specific karyotypic damage; non-rearranged $b c r$; proliferative growth pattern in vitro; numerous circulating myelocytes; profound thrombocytopenia preventing chemotherapy, dysplastic features associated with ACC of all leucocytes, chiefly mature granulocytes; possibility of hepatosplenomegaly and, hereafter, lymphadenopathy with ACC of lymphocytes eventually appearing in the course of the disease.

We are grateful to Sylvie Maillard for typing the manuscript and Robert Perry for reviewing the English text.
1 Felman P, Bryon PA, Gentilhomme O, et al. The syndrome of abnormal chromatin clumping in leucocytes: a myelodysplastic disorder with proliferative features? $\mathrm{Br} \mathcal{F} \mathrm{Hae}$ matol 1988;70:49-54.

2 Invernizzi R, Custodi $P$, De Fazio $P$, et al. The syndrome of abnormal chromatin clumping in leucocytes: clinical and biological study of a case. Haematologica 1990;75: 532-6.

3 Brizard A, Huret JL, Lamotte F, et al. Three cases of myelodysplastic-myeloproliferative disorder with abnormal chromatin clumping in granulocytes. $\mathrm{Br} f \mathrm{Haematol}$ 1989;72:294-5.

4 Wiedermann LM, Karchi KK, Shivi MKK, et al. The correlation of breakpoint cluster region rearrangement and $\mathrm{p} 210 \mathrm{Phl} / \mathrm{abl}$ expression with morphological analysis of Ph-negative chronic myeloid leukemia and other myeloproliferative diseases. Blood 1988;71:349-55.

5 Morel P, Bryon PA, Guyon JM, et al. Hemopathie maligne de type aplastique avec anomalies nucléairs majeures des granulocytes. Sem Hôp (Paris) 1968;49:3026-8.

6 Gustke SS, Becker GA, Garancis JC, et al. Chromatin clumping in mature leucocytes: an hitherto unrecognized abnormality. Blood 1970;35:637-58.

7 Winter JN, Variakojis D, Gaynor ER, et al. Low-dose cytosine arabinoside (Ara-C) therapy in the cytosine arabinoside (Ara-C) therapy in the
myelodysplastic syndromes and acute leukemia. Cancer myelodysplastic

8 Weil SC, Rose VL. A variant myelodysplastic syndrome with multilineage pelgeroid chromatin. Am $\mathcal{F}$ Clin Pathol 1986;85:176-9.

9 Jaen A, Irriguible D, Milla F, et al. Abnormal chromatin clumping in leucocytes: a clue to a new subtype of myelodysplastic syndrome. Eur f Haematol 1990; 45:209-14.

10 Bizet M, Callat MP, Goasguen J, et al. Chronic myelomoncytic leukemia (CMML) with lymphodenopathy. F. In: Schmalz F, Muffi GJ, eds. Myelodysplastic syndromes. Berlin: Springer Verlag, 1992:140-6.

11 Tefferi A, Hoagland HC, Therneau TM, et al. Chronic myelomonocytic leukemia: natural history and prognostic myelomonocytic leukemia: natural history and progn

12 Scott RB. Leukaemia (chronic myeloid leukaemia). Lancet 1957;i:1099-103.

13 Bennett JM, Catovsky D, Daniel MT, et al. Proposals for the classification of the myelodysplastic syndromes. $\mathrm{Br} f$ Haematol 1982;51:189-99.

14 Nowell PC. Cytogenetics of preleukemia. Cancer Genet Cytogenet 1982;5:265-78.

15 Prchal JT, Throckmorton DW, Carrol AJ, et al. A common progenitor for human myeloid and lymphoid cells. Nature 1978;274:590-1.

16 Raskind WH, Tirumali N, Jacobson R, et al. Evidence for a multistep pathogenesis of a myelodysplastic syndrome. Blood 1984;63:1318-23.

17 Abkowitz JL, Fialkow PJ, Niebruge DJ, et al. Pancytocytopenia as a a clonal disorder of a multipotent cytocytopenia as a a clonal disorder of a multipoten

18 Kere J, Ruutu T, de la Chapelle A. Monosomy 7 in granulocytes and monocytes in myelodysplastic syndrome. N Engl ₹ Med 1987;316:499-503.

19 Wallis JP, Joyner MV. Acute myeloid leukaemia developing in a patient with longstanding untreated chronic lymphocytic leukaemia. Acta Haematol 1986;75:229-31.

20 Inoshita T. Acute lymphoblastic leukemia following myelodysplastic syndrome. Am $\mathcal{F}$ Clin Pathol 1985;84:233-7.

21 Stark AN, Scott CS, Bhatt B, et al. Myelodysplastic syndrome coexisting with acute lymphoblastic leukaemia. fyndrome coexisting with acute

22 Wajima T, Mukhopadhyay M. Co-existing myelodysplastic syndrome and malignant lymphoma with IgA monoclonal gammopathy terminated in acute myeloid leukemia shorgammopathy terminated in acute myeloid leukemia shor-
tly after treatment of malignant lymphoma and prostate tly after treatment of malignant lymp
cancer. Blood 1991;78(suppl 1):462a.

23 Copplestone JA, Mufti GJ, Hamblin TJ, et al. Immunological abnormalities in myelodysplastic syndromes. $\mathrm{Br} \mathcal{f}$ Haematol 1989;63:149-59.

24 Colombat PH, Renoux M, Lamaguère JP, et al. Immunologic indices in myelodysplastic syndromes. Cancer 1988;61:1075-81.

25 Bastion Y, Thomas X, Felman P, et al. High risk myelodysplastic syndrome coexistent with chronic lymphocytic leukemia for more than 9 years: inhibition of the myeloid clone by the lymphoid clone? Leukemia 1991;5:1006-9.

26 Provan AB, Majer RV, Smith AG, et al. Chronic myelomonocytic leukamia associated with T cell receptor $\delta$ gene ocytic leukamia associated with T cell recep
rearrangement. $\mathcal{F}$ Clin Pathol $1991 ; 44: 344-5$.

27 Griesinger F, Jansen B, Kersey JH. Differentiation in mature $T$, lymphoid leukemia cells is unstable and mature T lymphoid leukemia cells is unstable and
reversible to myeloid cells, without the involvement of a reversible to myeloid cells, without the involvement
common stem cell. $\mathcal{F}$ Immunol 1991;147:3336-41.

28 Boehm T, Rabbitts TH. A chromosomal basis of lymphoid malignancy in man. Eur $\mathcal{F}$ Biochem 1989;185:1-17.

29 Varma N, Dash S, Marwaha N, et al. A case of myelodysplastic syndrome with abnormal chromatin clumping in leucocytes. Br $\mathcal{F}$ Haematol 1989;73:135.

30 Linman JW, Bagby GC. The preleukemic syndrome (hemopoietic dysplasia). Cancer 1978;42:854-64. 